\title{
NOTE ON THE NEVANLINNA PROXIMITY FUNCTION
}

\author{
JOHN L. LEWIS
}

\begin{abstract}
Let $\lambda$ be a positive function on $(0, \infty)$ with $\lim _{r \rightarrow \infty} \lambda(r)=\infty$, and $A$ an arbitrary set of capacity zero. An example is given of a meromorphic function $f$ for which $m(r, a) \rightarrow \infty, r \rightarrow \infty$, whenever $a \in A$, and $T(r, f)=$ $O\left[(\log r)^{2} \lambda(r)\right], r \rightarrow \infty$.
\end{abstract}

1. Introduction. Given $f$ meromorphic in the complex plane, define $m(r, a)$, $N(r), n(r)$ and $T(r)$ in the usual way relative to $f$ (see for example [3]). In this paper we consider the following problem:

If $f$ is a meromorphic function satisfying certain growth restrictions, for how large a set $A$ can

$$
\lim _{r \rightarrow \infty} m(r, a)=\infty \text { whenever } a \in A .
$$

It is well known that $A$ must have capacity zero. We note that the corresponding problem for entire functions of a given order has been completely solved by Drasin and Weitsman [2]. In [1] Damodaran proved

THEOREM. Let $A$ be an arbitrary set of capacity zero. Given $\lambda(r)$ tending to infinity as $r \rightarrow \infty$, there exists a meromorphic function which satisfies (1.1) and such that

$$
T(r)=O\left(\lambda(r)(\log r)^{3}\right), \quad r \rightarrow \infty .
$$

Moreover, for any meromorphic function satisfying

$$
T(r)=O\left((\log r)^{2}\right), \quad r \rightarrow \infty,
$$

the set $A$ can have at most one element.

The above theorem leaves open the precise requirements on $A$ for orders of growth between $O\left[(\log r)^{2}\right]$ and $O\left[\lambda(r)(\log r)^{3}\right]$. In this note we complete the solution of the above problem by showing

THEOREM 1. Let $\lambda$ and $A$ be as in the previous theorem. Then there exists a meromorphic function $f$ which satisfies (1.1) and such that

$$
T(r)=O\left[\lambda(r)(\log r)^{2}\right], \quad r \rightarrow \infty .
$$

2. Proof of Theorem 1. We may assume that $\lambda$ is nondecreasing, absolutely continuous, $\lambda \geqslant 1$, and $r^{-1 / 2} \lambda(r)$ nonincreasing for $r \geqslant 1$. Otherwise we

Received by the editors March 17, 1977 and, in revised form, August 29, 1977.

AMS (MOS) subject classifications (1970). Primary 30A70; Secondary 30A44.

Key words and phrases. Meromorphic function, Nevanlinna proximity function, capacity. 
replace $\lambda$ by $\lambda_{1}$, where $\lambda_{1}$ has these properties, and $\lambda_{1} \leqslant \lambda$ for sufficiently large $r$. Put

$$
\sigma(z)=z \int_{0}^{\infty} \lambda^{\prime}\left(\frac{t}{16}\right)(t+z)^{-1} d t .
$$

Using the fact that $r^{-1 / 2} \lambda(r)$ is nonincreasing, it follows easily that

$$
k_{1} \lambda(r / 16) \leqslant|\sigma(z)| \leqslant k_{2} \lambda(r / 16) \text {, }
$$

when $|z|=r, r \geqslant 16$, and $|\arg z| \leqslant \pi / 2$. Here $k_{1}$ and $k_{2}$ are positive constants.

Let $\left(\nu_{n}\right)_{1}^{\infty}$ be a nondecreasing sequence of positive integers. Given positive integers $k$ and $n$, where $1 \leqslant k \leqslant \nu_{n}$, let

$$
\begin{aligned}
& E_{k, n}^{\prime}=\left\{z: \frac{7}{8} 2^{n} \leqslant|z| \leqslant \frac{9}{8} 2^{n+1},\left|\arg z-(4 k-1) / 16 v_{n}\right| \leqslant 1 / 12 v_{n}\right\}, \\
& E_{k, n}=\left\{z: 2^{n} \leqslant|z| \leqslant 2^{n+1},\left|\arg z-(4 k-1) / 16 v_{n}\right|<1 / 24 v_{n}\right\}
\end{aligned}
$$

when $n$ is even, and

$$
\begin{aligned}
& E_{k, n}^{\prime}=\left\{z: \frac{7}{8} 2^{n} \leqslant|z| \leqslant \frac{9}{8} 2^{n+1},\left|\arg z+(4 k-1) / 16 v_{n}\right| \leqslant 1 / 12 v_{n}\right\}, \\
& E_{k, n}=\left\{z: 2^{n} \leqslant|z| \leqslant 2^{n+1},\left|\arg z+(4 k-1) / 16 v_{n}\right| \leqslant 1 / 24 v_{n}\right\}
\end{aligned}
$$

when $n$ is odd.

The proof of Theorem 1 is based upon the following lemma.

LEMMA 1. Let $\zeta_{k, n}, 1 \leqslant k \leqslant \nu_{n}, n=1,2, \ldots$, be a sequence of complex numbers with $\left|\zeta_{k, n}\right| \leqslant \sqrt{\sigma\left(2^{n}\right)}, n=1,2, \ldots$ If $\nu_{n} \leqslant \log \sigma\left(2^{n}\right), n=1,2, \ldots$, then there exist a meromorphic function $f$ and a positive integer $n_{0}$ such that

$$
\left|f(z)-\zeta_{k, n}\right| \leqslant \sigma\left(2^{n}\right)^{-1 / 4}, \quad z \in E_{k, n},
$$

for $1<k \leqslant \nu_{n}$ and $n \geqslant n_{0}$. Moreover,

$$
T(r)=O\left[\lambda(r)(\log r)^{2}\right], \quad r \rightarrow \infty .
$$

Proof. Let $k, n$, be positive integers, $1 \leqslant k \leqslant \nu_{n}$, and put $d_{n}=$ $2^{n} /\left(1000 \nu_{n}\right)$. It is easily seen that $\partial E_{k, n}^{\prime}$ can be covered by no more than $10002^{n} / \dot{d}_{n}$ disks:

$$
\Delta\left(\zeta_{m}, r_{m}\right)=\left\{\zeta:\left|\zeta-\zeta_{m}\right| \leqslant r_{m}\right\}, \quad \zeta_{m} \in \partial E_{k, n}^{\prime},
$$

with disjoint interiors and $r_{m} \leqslant d_{n} / 4$. Let

$$
Q(\zeta, z)=-\sum_{p=0}^{N}\left(\zeta-\zeta_{m}\right)^{p}\left(z-\zeta_{m}\right)^{-(p+1)}
$$

for $\zeta$ in $\Delta\left(\zeta_{m}, r_{m}\right)$, where $N=N(n)$ is to be specified later. We note that

$$
|Q(\zeta, z)| \leqslant(N+1)\left(d_{n}\right)^{N}\left|z-\zeta_{m}\right|^{-(N+1)}
$$

when $\left|z-\zeta_{m}\right| \leqslant d_{n}$, and $\zeta$ is in $\Delta\left(\zeta_{m}, r_{m}\right)$. Also

$$
\left|Q(\zeta, z)-(\zeta-z)^{-1}\right| \leqslant 4^{-N}\left|z-\zeta_{m}\right|^{-1},
$$

when $\left|z-\zeta_{m}\right| \geqslant d_{n}$, and $\zeta$ in $\Delta\left(\zeta_{m}, r_{m}\right)$. We remark that for fixed $z, Q(\cdot, z)$ is 
well defined on $\partial E_{k, n}^{\prime}$, except for a finite number of $\zeta \in \partial E_{k, n}^{\prime}$, since the disks $\Delta\left(\zeta_{m}, r_{m}\right)$ have disjoint interiors. Put

$$
\begin{aligned}
& g_{k, n}(z)=\frac{1}{2 \pi i} \int_{\partial E_{k, n}^{\prime}} \sigma(\zeta) Q(\zeta, z) d \zeta, \\
& h_{k, n}(z)=\frac{1}{2 \pi i} \int_{\partial E_{k, n}^{\prime}} \sigma(\zeta)(\zeta-z)^{-1} d \zeta .
\end{aligned}
$$

Let $\xi_{k, n}$ be a sequence of complex numbers with $\left|\xi_{k, n}\right| \leqslant \sqrt{\sigma\left(2^{n}\right)}$ for $1 \leqslant k$ $\left\langle\nu_{n}\right.$ and $n=1,2, \ldots$ In the sequel $c$ denotes a positive constant, not necessarily the same at each occurrence. Given $z$ with $|z| \geqslant 64$, choose $p$ a positive integer so that $2^{p}<|z|<2^{p+1}$. If $n<p-3$, then since $\zeta_{m}$ is in $\partial E_{k, n}^{\prime}$, we have $\left|\zeta_{m}\right|<2^{n+2}<|z| / 2$. Consequently,

$$
\left|z-\zeta_{m}\right| \geqslant|z| / 2 \geqslant 2^{p-1} \geqslant d_{n} .
$$

Using (2.3) it follows that

$$
\begin{aligned}
& \left|g_{k, n}(z)-h_{k, n}(z)\right| \leqslant c 2^{n} \sigma\left(2^{n+2}\right) 4^{-N} \sum_{m}\left|z-\zeta_{m}\right|^{-1} \\
& \leqslant c 2^{n-p_{\nu_{n}}} 4^{-N_{\sigma}}\left(2^{n+2}\right)
\end{aligned}
$$

since there are at most $c v_{n}$ terms in the sum and each is $\leqslant 2^{1-p}$. We now let $N(n)=\left[5 \log \sigma\left(2^{n+2}\right)\right]+1$, where [ ] denotes the greatest integer function. Clearly, $4^{-N} \sigma\left(2^{n+2}\right)^{5}<1$. Since $\nu_{n} \leqslant \log \sigma\left(2^{n}\right)$, and $\left|\xi_{k, n}\right|<\sqrt{\sigma\left(2^{n}\right)}$, it follows from (2.4) that

$$
\begin{aligned}
& \sum_{n=1}^{p-3} 2^{-n / 2}\left(\sum_{k=1}^{\nu_{n}}\left|\xi_{k, n}\right|\left|g_{k, n}(z)-h_{k, n}(z)\right|\right) \\
&<c \sum_{n=1}^{p-3} 2^{-n / 2}\left(2^{n-p}\right) \leqslant c 2^{-p / 2}
\end{aligned}
$$

Now if dist $\left(z, \partial E_{k, n}^{\prime}\right) \geqslant d_{n}$ for each $n \geqslant p-2$ and $1<k<\nu_{n}$, then from (2.3) we have

$$
\begin{aligned}
\left|g_{k, n}(z)-h_{k, n}(z)\right| & \leqslant c 2^{n} \sigma\left(2^{n+2}\right)\left(4^{N} d_{n}\right)^{-1} \\
& \leqslant c \nu_{n} \sigma\left(2^{n+2}\right) 4^{-N} .
\end{aligned}
$$

Hence,

$$
\begin{array}{r}
\sum_{n=(p-2)}^{\infty} 2^{-n / 2}\left(\sum_{k=1}^{v_{n}}\left|\xi_{k, n}\right|\left|g_{k, n}(z)-h_{k, n}(z)\right|\right) \\
\leqslant c \sum_{n=p-2} 2^{-n / 2} \leqslant c 2^{-p / 2}
\end{array}
$$

Let 


$$
\begin{gathered}
F(z)=\sum_{n=1}^{\infty} 2^{-n / 2}\left(\sum_{k=1}^{\nu_{n}} \zeta_{k, n} g_{k, n}(z)\right), \quad G(z)=\sum_{n=1}^{\infty} 2^{-n / 2}\left(\sum_{k=1}^{\nu_{n}} g_{k, n}(z)\right), \\
f(z)=F(z) / G(z) .
\end{gathered}
$$

The series defining $F$ and $G$ converge uniformly on compact subsets of the complex plane, as follows from (2.5), (2.7), and the fact that $h_{k, n}(z)=0$, for $z$ in a compact subset, except for a finite number of pairs $(k, n)$.

If $z \in E_{j, p}$, then

$$
\left|f(z)-\zeta_{j, p}\right|=|G(z)|^{-1}\left|F(z)-\zeta_{j, p} G(z)\right|
$$

and dist $\left(z, \partial E_{k, n}^{\prime}\right) \geqslant d_{n}$ for $1 \leqslant k \leqslant \nu_{n}, n=1,2, \ldots$ Since $h_{k, n}(z)=0$ when $(k, n) \neq(j, p)$, and $h_{j, p}(z)=\sigma(z), z \in E_{j, p}$, it follows from (2.5) and (2.7) with $\left|\xi_{k, n}\right|=1$, that $\left|G(z)-2^{-p / 2} \sigma(z)\right| \leqslant c 2^{-p / 2}$. Hence,

$$
|G(z)| \geqslant 2^{-p / 2}[|\sigma(z)|-c], \quad z \in E_{j p} .
$$

Also,

$$
\begin{aligned}
\left|F(z)-\zeta_{j, p} G(z)\right| \leqslant & \sum_{\substack{n=1 \\
n \neq p}}^{\infty}\left(\sum_{k=1}^{\nu_{n}}\left|\zeta_{k, n}-\zeta_{j, p}\right|\left|g_{k, n}(z)\right|\right) 2^{-n / 2} \\
& +2^{-p / 2} \sum_{\substack{k=1 \\
k \neq j}}^{\nu_{p}}\left|\zeta_{k, p}-\zeta_{j, p}\right|\left|g_{k, p}(z)\right| .
\end{aligned}
$$

Using (2.5) and (2.7), we get

$$
\left|F(z)-\zeta_{j, p} G(z)\right| \leqslant c\left(\left|\zeta_{j, p}\right|+1\right) 2^{-p / 2} \leqslant c \sqrt{\sigma\left(2^{p}\right)} 2^{-p / 2} .
$$

From (2.8) and (2.1), we now conclude that

$$
\left|f(z)-\zeta_{j, p}\right| \leqslant \frac{c\left(\sqrt{\sigma\left(2^{p}\right)}\right) 2^{-p / 2}}{2^{-p / 2}(|\sigma(z)|-c)} \leqslant \sigma\left(2^{p}\right)^{-1 / 4}
$$

whenever $z \in E_{j, p}$ and $p \geqslant n_{0}$.

To conclude the proof of Lemma 1 , we estimate $T(r, f)$. Since $T(r, f) \leqslant$ $T(r, F)+T(r, 1 / G) \leqslant T(r, F)+T(r, G)+O(1)$, it suffices to estimate $T(r, F)$ and $T(r, G)$. Recall for given positive integers $k, n, 1 \leqslant k \leqslant \nu_{n}$, that $\partial E_{k, n}^{\prime}$ was covered by at most $c 2^{n} / d_{n}=c \nu_{n}$ disks $\Delta\left(\zeta_{m}, r_{m}\right)$. Moreover, $g_{k, n}$ has a pole of order $N(n)+1 \leqslant 6 \log \sigma\left(2^{n+2}\right)$, at $\zeta_{m}$. Since $\nu_{n} \leqslant \log \sigma\left(2^{n}\right)$, it follows that $g_{k, n}$ has at most $c\left[\log \sigma\left(2^{n+2}\right)\right]^{2}$ poles all in $\left\{z: 2^{n-1} \leqslant|z| \leqslant\right.$ $\left.2^{n+2}\right\}$. If $2^{p} \leqslant r<2^{p+1}$, it follows from (2.1) that

$$
\begin{aligned}
n(r, F) & =n(r, G) \leqslant c \sum_{n=1}^{p+2}\left[\log \sigma\left(2^{n+2}\right)\right]^{2} \nu_{n} \leqslant c p\left[\log \sigma\left(2^{p+4}\right)\right]^{3} \\
& \leqslant c(\log r)(\log \lambda(r))^{3} \leqslant c(\log r) \lambda(r) .
\end{aligned}
$$

Hence, 


$$
N(r, F)=N(r, G)<c(\log r)^{2} \lambda(r) .
$$

To estimate $m(r, F)$, we note from (2.5) and (2.7) for a given $z$ with $2^{p}<|z|<2^{p+1}$, that

$$
|F(z)|<c+\sum_{n=p-2}^{p+2} \sum_{k=1}^{\nu_{n}}\left|\zeta_{k, n}\right|\left|g_{k, n}\right|(z) .
$$

Since $\left|\zeta_{k, n}\right|<\sqrt{\sigma\left(2^{n+2}\right)}$, it follows that

$$
\log ^{+}|F(z)| \leqslant c+\log \sigma\left(2^{p+4}\right)+\log \nu_{p+2}+\sum_{n=p-2}^{p+2} \sum_{k=1}^{v_{n}} \log ^{+}\left|g_{k, n}\right|(z) .
$$

Hence, for $2^{p}<r<2^{p+1}$,

$$
m(r, F)<c+2 \log ^{+} \sigma\left(2^{p+4}\right)+\sum_{n=p-2}^{p+2} \sum_{k=1}^{\nu_{n}} m\left(r, g_{k, n}\right) .
$$

To estimate $m\left(r, g_{k, n}\right), 2^{p} \leqslant r<2^{p+1}$, we note from the definition of $g_{k, n}$ that $g_{k, n}(z)=\Sigma_{m} R_{m}(z)$, where

$$
R_{m}(z)=\frac{1}{2 \pi i} \int_{\Delta\left(\zeta_{m}, r_{m}\right) \cap \partial E_{k, n}^{\prime}} \sigma(\zeta) Q(\zeta, z) d \zeta,
$$

and the sum has at most $c v_{n}$ terms. From (2.2) we have

$$
\left|R_{m}(z)\right| \leqslant c N 2^{n} \sigma\left(2^{n+2}\right)\left(d_{n}\right)^{N}\left|z-\zeta_{m}\right|^{-(N+1)},
$$

when $\left|z-\zeta_{m}\right| \leqslant d_{n}$ and from (2.3), $\left|R_{m}(z)\right| \leqslant c \sigma\left(2^{n+2}\right) d_{n}$, when $\left|z-\zeta_{m}\right| \geqslant$ $d_{n}$. Hence,

$$
\begin{aligned}
\log ^{+}\left|R_{m}(z)\right| \leqslant c & +\log N+n \log 2+2 \log \sigma\left(2^{n+2}\right) \\
& +(N+1) \log d_{n}+(N+1) \log ^{+}\left(1 /\left|z-\zeta_{m}\right|\right) .
\end{aligned}
$$

Since $N<6 \log \sigma\left(2^{n+2}\right)$ and $d_{n}<2^{n}$, it follows that

$$
m\left(r, R_{m}\right) \leqslant c n \log \sigma\left(2^{n+2}\right) .
$$

Consequently,

$$
\begin{aligned}
m\left(r, g_{k, n}\right) & =m\left(r, \sum_{m} R_{m}\right) \leqslant c \nu_{n}+\sum_{m} m\left(r, R_{m}\right) \\
& \leqslant c \nu_{n}+c\left(\nu_{n}\right) n \log \sigma\left(2^{n+2}\right) \leqslant c\left[\log \sigma\left(2^{n+2}\right)\right]^{2} n .
\end{aligned}
$$

Using (2.10), we conclude

$$
m(r, F) \leqslant c p\left[\log \sigma\left(2^{p+4}\right)\right]^{3} \leqslant c(\log r) \lambda(r) .
$$

Similarly, $m(r, G) \leqslant c(\log r) \lambda(r)$. From (2.9) we now deduce

$$
\max [T(r, F), T(r, G)] \leqslant c(\log r)^{2} \lambda(r) .
$$

This completes the proof of Lemma 1. Theorem 1 now follows from Lemma 1 and an argument of Drasin and Weitsman [2, \$7]. We sketch their proof when $A$ is a compact set of capacity zero. In this case there is a logarithmic potential $P$ of the form: 


$$
P(z)=-\sum_{j=1}^{\infty} \alpha_{j} \log \left|z-a_{j}\right|,
$$

which is infinite on $A$. Here $a_{j} \in A, j=1,2, \ldots,\left(a_{i} \neq a_{j}, i \neq j\right)$, and $\sum_{j=1}^{\infty} \alpha_{j}=1$. Let $f$ be as in Lemma 1. Then it is possible to choose the sequences $\left(\nu_{n}\right)$ and $\left(\zeta_{k, n}\right)$ in Lemma 1 so that for each positive integer $j$ and $\varepsilon_{j}>0$, there corresponds an $r_{j}=r_{j}\left(\varepsilon_{j}\right)$ with the following property:

$$
\left|f\left(r e^{i \theta}\right)-a_{j}\right| \leqslant \varepsilon_{j}, \quad \theta \in E_{j}(r), r \geqslant r_{j},
$$

where $E_{j}(r)$ has Lebesgue measure $\geqslant c \alpha_{j}$. Given $N$ a positive integer, choose $\varepsilon_{j}, 1 \leqslant j \leqslant N$, so small that the corresponding sets $E_{j}(r)$ are disjoint for $r \geqslant \max \left\{r_{j}: 1 \leqslant j \leqslant N\right\}$. If $r \geqslant \max \left\{r_{j}: 1 \leqslant j \leqslant N\right\}$,

$$
\begin{aligned}
2 \pi m(r, a) & \geqslant \sum_{j=1}^{N} \int_{E_{j}(r)} \log ^{+}\left|1 /\left(f\left(r e^{i \theta}\right)-a\right)\right| d \theta \\
& \geqslant c \sum_{j=1}^{N} \alpha_{j} \log ^{+}\left(1 /\left(\varepsilon_{j}+\left|a-a_{j}\right|\right)\right) .
\end{aligned}
$$

Letting first $r \rightarrow \infty$ through a certain sequence, and then $\varepsilon_{j} \rightarrow 0,1 \leqslant j \leqslant N$, we get

$$
2 \pi \liminf _{r \rightarrow \infty} m(r, a) \geqslant c \sum_{j=1}^{N} \alpha_{j} \log ^{+}\left|1 /\left(a-a_{j}\right)\right| .
$$

Letting $N \rightarrow \infty$, it follows for $a \in A$ that

$$
2 \pi \liminf _{r \rightarrow \infty} m(r, a) \geqslant c P(a)=+\infty .
$$

\section{REFERENCES}

1. M. Damodaran, On the distribution of values of meromorphic functions of slow growth, Complex Analysis, Springer Lecture Notes in Math., vol. 599, Springer-Verlag, New York, 1977.

2. D. Drasin and A. Weitsman, The growth of the Nevanlinna proximity function and the logarithmic potential, Indiana Univ. Math. J. 20 (1971), 699-715.

3. W. K. Hayman, Meromorphic functions, Clarendon Press, Oxford, 1964.

Department of Mathematics, University of Maryland, College Park, Maryland 20742 